OPEN ACCESS

Edited by:

Sandra Donnini,

University of Siena, Italy

Reviewed by:

Valentina Citi,

University of Pisa, Italy

Lara Testai,

University of Pisa, Italy

Zhongjian Cheng,

Temple University, United States

*Correspondence:

Honggang Wang

whg197167@vip.henu.edu.cn

Specialty section:

This article was submitted to

Cellular Biochemistry

a section of the journal

Frontiers in Cell and Developmental

Biology

Received: 09 November 2020

Accepted: 04 January 2021

Published: 18 February 2021

Citation:

Zhao S, Li X, Li X, Wei $X$ and Wang H (2021) Hydrogen Sulfide Plays an Important Role in Diabetic Cardiomyopathy.

Front. Cell Dev. Biol. 9:627336

doi: $10.3389 / f c e l l .2021 .627336$

\section{Hydrogen Sulfide Plays an Important Role in Diabetic Cardiomyopathy}

\author{
Shizhen Zhao, Xiaotian Li, Xinping Li, Xiaoyun Wei and Honggang Wang* \\ Institute of Biomedical Informatics, Bioinformatics Center, School of Basic Medical Sciences, Henan University, Kaifeng, China
}

Diabetic cardiomyopathy is an important complication of diabetes mellitus and the main cause of diabetes death. Diabetic cardiomyopathy is related with many factors, such as hyperglycemia, lipid accumulation, oxidative stress, myocarditis, and apoptosis. Hydrogen sulfide $\left(\mathrm{H}_{2} \mathrm{~S}\right)$ is a newly discovered signal molecule, which plays an important role in many physiological and pathological processes. Recent studies have shown that $\mathrm{H}_{2} \mathrm{~S}$ is involved in improving diabetic cardiomyopathy, but its mechanism has not been fully elucidated. This review summarizes the research on the roles and mechanisms of $\mathrm{H}_{2} \mathrm{~S}$ in diabetic cardiomyopathy in recent years to provide the basis for in-depth research in the future.

Keywords: hydrogen sulfide, diabetic, cardiovascular diseases, diabetic cardiomyopathy, diabetic vascular diseases

\section{INTRODUCTION}

Diabetes has reached epidemic levels, and its prevalence has increased significantly in recent years. In the 1990s, the number of people with diabetes worldwide was $\sim 135$ million, and this figure could reach 300 million by 2025. Cardiomyopathy is an important complication of diabetes, and more than $50 \%$ of diabetic patients die of cardiomyopathy (Balakumar et al., 2016; Htay et al., 2019). Diabetic cardiomyopathy (DCM) is a disease that leads to myocardial dysfunction without major risk factors (including hypertension or coronary artery disease) (Jia et al., 2018b). DCM is characterized by various forms, including hypertrophy of cardiomyocytes, reduction of myofibrils, interstitial fibrosis, and myocardial microvascular lesions (Russo and Frangogiannis, 2016; Varma et al., 2018). It has been reported that hyperglycemia, lipid accumulation, oxidative stress, inflammation, calcium overload, mitochondrial dysfunction, myocarditis, and apoptosis might be related with the pathogenesis of DCM (Jia et al., 2018a; Dillmann, 2019). However, the mechanism of DCM has not been fully studied. Therefore, it is particularly important to explore the pathogenesis and treatments of diabetic cardiovascular diseases. It has been reported that hydrogen sulfide $\left(\mathrm{H}_{2} \mathrm{~S}\right)$ has many biological functions (Sun H. J. et al., 2019) and is involved in many diseases (Wang et al., 2020a), including diabetes-related diseases (Qian et al., 2018). This review summarizes the research about the roles and mechanisms of $\mathrm{H}_{2} \mathrm{~S}$ in DCM in recent years to provide the basis for in-depth research in the future.

\section{OVERVIEW OF DCM}

The term diabetic cardiomyopathy was first used by Rubler in 1972. DCM increased the risk of heart failure in diabetes by four to five times. It is characterized by myocardial cell loss and myocardial fibrosis, ventricular systolic and/or diastolic dysfunction, without significant coronary atherosclerosis and hypertension (Poornima et al., 2006; Karbasforooshan and Karimi, 2017; Yao et al., 2018). In the early stage of DCM, the pathological alterations are mainly 
in the myocardial interstitium, including the formation of nonenzymatic advanced glycation end products (AGEs), impaired compliance, and ischemic intravascular diseases. Although the morphology of myocardial cells and small coronary arteries has been preserved anatomically, these changes still lead to impaired myocardial contractility. With the development of the disease, left ventricular (LV) hypertrophy is characterized by hypertrophy of myocardial cells, interstitial fibrosis and perivascular fibrosis, thickening of the capillary basement membrane, and formation of intracapillary microaneurysms (Felicio et al., 2016; Jia et al., 2018a). DCM occurs in both type 1 and type 2 diabetes mellitus (Sulaiman et al., 2010), affects nearly two-thirds of diabetic patients, and is the leading cause of diabetes-related morbidity and mortality (Boudina and Abel, 2007; Tillquist and Maddox, 2012). Although the strict control of blood glucose seems to play an important role in the prevention and treatment of DCM, there is an urgent need for new methods and specific therapeutic agents for DCM. Therefore, it is necessary to understand the pathogenesis of DCM (Acar et al., 2011). The molecular mechanism of DCM may be multifactorial, including but not limited to inflammation, cell death, nitroso stress, oxidative stress, impairment in calcium handling, increased AGEs, and mitochondrial dysfunction (Al Hroob et al., 2019). These pathologic alterations in cardiomyocytes are primarily triggered by many metabolic disorders including hyperglycemia, dyslipidemia, increased free fatty acid (FFA) release, and insulin resistance (Mahmoud, 2017). However, the pathogenesis of DCM remains to be elucidated.

\section{OVERVIEW OF $\mathrm{H}_{2} \mathrm{~S}$}

For many years, $\mathrm{H}_{2} \mathrm{~S}$ has been considered to be a toxic and odorous gas. However, since the 1990s, many studies have shown that $\mathrm{H}_{2} \mathrm{~S}$, along with $\mathrm{NO}$ and $\mathrm{CO}$, belongs to the category of gasotransmitters (Wang et al., 2020b). Three "classic" $\mathrm{H}_{2} \mathrm{~S}$ producing enzymes have been identified: cystathionine- $\gamma$-lyase (CSE), cystathionine- $\beta$-synthase (CBS), and 3-mercaptopyruvate thiotransferase (3-MST) (Rose et al., 2017). The expression of $\mathrm{H}_{2} \mathrm{~S}$-producing enzyme is subcellular and tissue-specific. At the cellular level, CSE occurs strictly in the cytoplasm, while cysteine aminotransferase (CAT) is located in the mitochondria. In terms of tissue specificity, CSE is the most abundant in the cardiovascular system, while CBS is dominant in the nervous system and liver and is expressed in the heart (Kar et al., 2019; Shen et al., 2019). In the process of endogenous $\mathrm{H}_{2} \mathrm{~S}$ production, CBS catalyzes the $\beta$-substitution reaction of homocysteine with serine to produce L-cystathionine. L-cystenine is produced by the elimination of $\alpha, \gamma$-cysteine of L-cystathionine catalyzed by CSE. L-cystenine then produces $\mathrm{H}_{2} \mathrm{~S}$ via $\beta$ elimination reaction catalyzed by CSE/CBS. L-cystenine also produces 3mercaptopyruvate (3-MP) by transferring its amines to $\alpha$ ketoglutarate catalyzed by CAT. 3-MST catalyzes the sulfur of 3-MP to convert into $\mathrm{H}_{2} \mathrm{~S}$. In cardiomyocytes that mainly express $\mathrm{CSE}, \mathrm{H}_{2} \mathrm{~S}$ is produced with L-cysteine as substrate under the catalysis of CSE (Figure 1) (Behera et al., 2019). In addition, there are some other recognized or assumed sources of $\mathrm{H}_{2} \mathrm{~S}$, including
D-amino acid oxylase (Han et al., 2020) and methionine oxidase (Pol et al., 2018). In biological systems, several non-enzymatic methods can also produce $\mathrm{H}_{2} \mathrm{~S}$ (Yang et al., 2019). In T2DM patients with DCM, endogenous $\mathrm{H}_{2} \mathrm{~S}$ production by CSE is inhibited in cardiomyocytes (Mard et al., 2016). $\mathrm{H}_{2} \mathrm{~S}$ level in plasma is decreased, and the supplement of $\mathrm{H}_{2} \mathrm{~S}$ can reduce the cardiomyopathy dysfunction induced by hyperglycemia (Kar et al., 2019).

$\mathrm{H}_{2} \mathrm{~S}$ can act as a signal molecule immediately after it is released, and it can also be stored as bound endosulfan, which can release $\mathrm{H}_{2} \mathrm{~S}$. At physiological $\mathrm{pH}$, nearly two-thirds of $\mathrm{H}_{2} \mathrm{~S}$ is in the form of hydrogen sulfide anion (HS-) (Guo et al., 2016). $\mathrm{H}_{2} \mathrm{~S}$ plays important roles in many physiological processes, including vasodilation, blood pressure reduction (Greaney et al., 2017; Jin et al., 2017), anti-apoptosis (Li et al., 2019b), anti-inflammation (Zhao et al., 2019), antioxidative stress (Tocmo and Parkin, 2019), cell survival/death, cell differentiation, cell proliferation/hypertrophy, mitochondrial bioenergetics/biogenesis, and endoplasmic reticulum stress (ERS) (Figure 1) (Zhang D. et al., 2017). Recent studies have shown that $\mathrm{H}_{2} \mathrm{~S}$ ameliorates diabetic complications including endothelial dysfunction (Li et al., 2019a), nephropathy (Karmin and Siow, 2018), retinopathy (Wang P. et al., 2019), and cardiovascular diseases (Citi et al., 2018). Research has reported that in diabetes, intraislet $\mathrm{H}_{2} \mathrm{~S}$ could promote opening of the ATP dependent potassium channel, increase $\mathrm{K}^{+}$efflux to lead to cell membrane hyperpolarization, and then close the L-type voltagedependent calcium channel, thus inhibiting insulin secretion of pancreatic beta cells. On the contrary, much literature has shown that $\mathrm{H}_{2} \mathrm{~S}$ can promote the release of insulin from $\beta$ cells. The reason for the above contradiction has not been fully studied (Szabo, 2012; Piragine and Calderone, 2020). In addition, $\mathrm{H}_{2} \mathrm{~S}$ could also regulate the apoptosis of islet beta cells and increase ERS and apoptosis of pancreatic beta cells by inhibiting the extracellular signal regulated kinase (ERK) and activating p38 mitogen activated protein kinase (p38 MAPK) signal pathway (Yang et al., 2007). Other studies have shown that $\mathrm{H}_{2} \mathrm{~S}$ could inhibit high glucose (HG)-induced apoptosis of pancreatic beta cells through the antioxidant, anti-inflammatory, or protein kinase B (Akt) signaling pathways (Taniguchi et al., 2011). However, the mechanism of $\mathrm{H}_{2} \mathrm{~S}$ in diabetes is not fully understood.

\section{THE ROLE OF $\mathrm{H}_{2} \mathrm{~S}$ IN DCM \\ Exogenous $\mathrm{H}_{2} \mathrm{~S}$ Improves DCM by Inhibiting Oxidative Stress, Inflammation, and Apoptosis}

One of the important pathophysiological factors of DCM is that persistent hyperglycemia induces oxidative stress and apoptosis to lead to cardiac fibrosis (Boudina and Abel, 2007). It has been reported that blocking the formation of reactive oxygen species (ROS) inhibited the expression of the fibrosis-related factor matrix metalloproteinase-2 (MMP-2) induced by HG (Yamagishi et al., 2001). Exogenous $\mathrm{H}_{2} \mathrm{~S}$ can suppress oxidative stress by inhibiting ROS production (Xiao et al., 2018), which may be the 


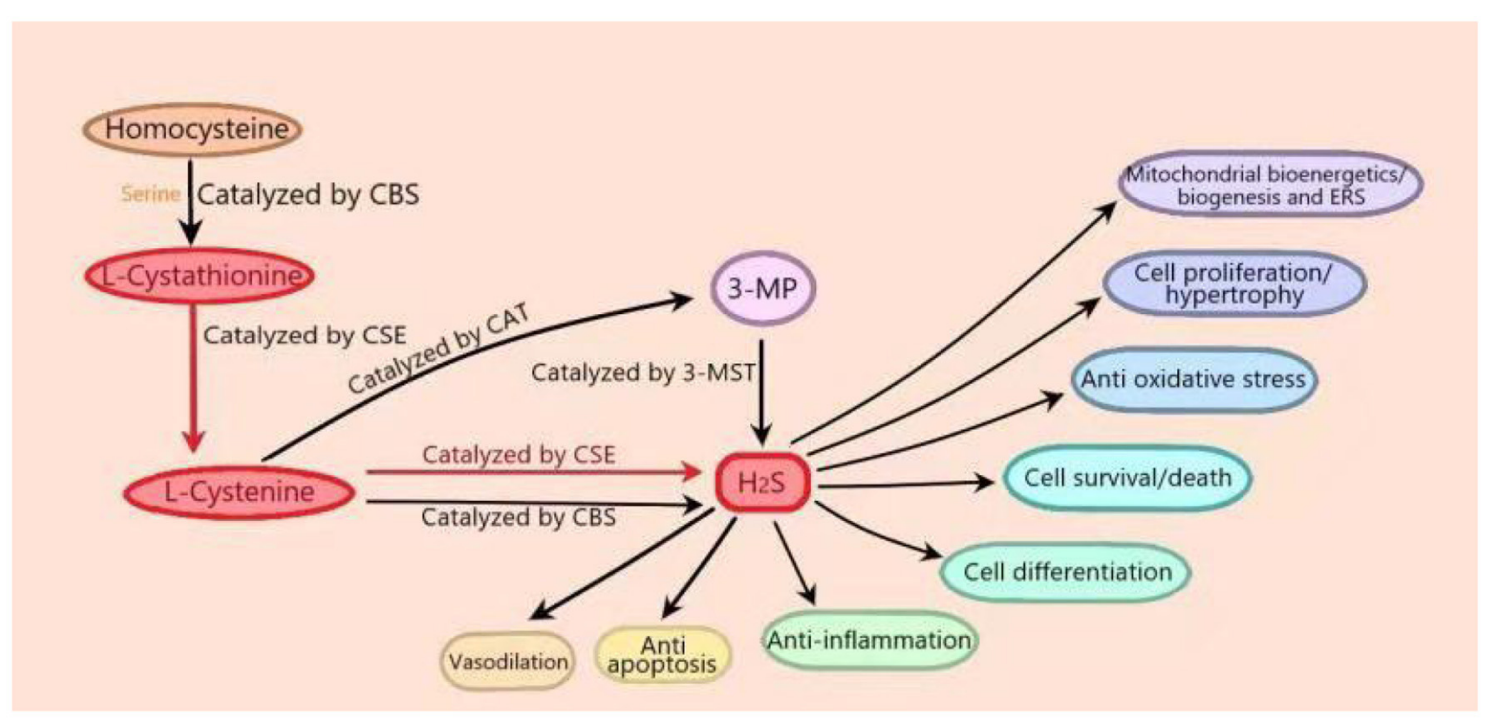

FIGURE 1 | In vivo synthesis process and biological function of hydrogen sulfide $\left(\mathrm{H}_{2} \mathrm{~S}\right)$. In the process of endogenous $\mathrm{H}_{2} \mathrm{~S}$ production, firstly,

cystathionine- $\beta$-synthase (CBS) catalyzes the $\beta$-substitution reaction of homocysteine with serine to produce L-cystathionine. L-cystenine is produced by the elimination of $\alpha, \gamma$-cysteine of L-cystathionine catalyzed by cystathionine- $\gamma$-lyase (CSE). L-cystenine then produces hydrogen sulfide $\left(\mathrm{H}_{2} \mathrm{~S}\right)$ via $\beta$ elimination reaction catalyzed by CSE/CBS. L-cystenine also produces 3-mercaptopyruvate (3-MP) by transferring its amines to $\alpha$-ketoglutarate catalyzed by cysteine aminotransferase (CAT). 3-Mercaptopyruvate thiotransferase (3-MST) catalyzes the sulfur of 3-MP to convert into $\mathrm{H}_{2} \mathrm{~S}$. In cardiomyocytes that mainly express CSE, $\mathrm{H}_{2} \mathrm{~S}$ is produced with L-cysteine as substrate under the catalysis of CSE. (The part in the figure is marked in red.) $\mathrm{H}_{2} \mathrm{~S}$ plays important roles in many physiological processes, including vasodilation, blood pressure reduction, anti-apoptosis, anti-inflammation, anti-oxidative stress, cell survival/death, cell differentiation, cell proliferation/hypertrophy, mitochondrial bioenergetics/biogenesis, and endoplasmic reticulum stress.

mechanism of its anti-diabetic myocardial fibrosis. Nuclear factor kappa-B (NF- $\mathrm{k}$ ) is a ubiquitous inducible transcription factor. Activation of NF- $\mathrm{kB}$ can upregulate the expression of fibrosisrelated factors, including transforming growth factor b1 (TGFb1) and MMP-2, and increase Fas-ligand (Fas-L) expression to induce Fas-L-mediated apoptosis in the myocardium of diabetic rats, which leads to myocardial fibrosis (Darville and Eizirik, 2001; Chen et al., 2003; Hein et al., 2003). NaHS (a donor of $\mathrm{H}_{2} \mathrm{~S}$ ) treatment may play an anti-fibrogenic role by reducing HG-induced expression of NF- $\mathrm{kB}$ and the fibrogenic factor such as TGF-b1 and MMP-2 to improve diabetic myocardial fibrosis (El-Seweidy et al., 2011). Therefore, it can be inferred that $\mathrm{H}_{2} \mathrm{~S}$ ameliorates DCM by inhibiting oxidative stress and apoptosis through suppressing the NF- $\mathrm{KB}$ pathway and ROS production.

Except for oxidative stress and apoptosis, inflammation is involved in the pathogenesis of DCM (Al-Rasheed et al., 2017; Zhao et al., 2017; Zou et al., 2019). A chronic, low-level state of systemic and sterile inflammation is an important feature of DCM (Sharma et al., 2018). The NLRP3 inflammasome is an important complex protein that mediates inflammation (Wang H. et al., 2019). Studies showed that suppressing NLRP3 notably improved DCM (Ye et al., 2017). $\mathrm{H}_{2} \mathrm{~S}$ decreases HGinduced cell apoptosis, ROS production, and the expression of the NLRP3 inflammasome, Toll-like receptor 4 (TLR4), and NF$\kappa B$. NLRP3 gene silencing improves HG-induced cell apoptosis and inflammation, and the TLR4/NF- $\kappa B$ pathway mediates the activation of NLRP3 induced by HG in cardiac cells. These results indicate that $\mathrm{H}_{2} \mathrm{~S}$ improves DCM by inhibiting HG-induced cardiomyocyte inflammation and apoptosis by suppressing the
NLRP3 inflammasome through inhibiting the TLR4/NF- $\mathrm{BB}$ pathway (Huang et al., 2016). The NLRP3 inflammasome will be an important target for $\mathrm{H}_{2} \mathrm{~S}$ to improve DCM. LV dysfunction, myocardial hypertrophy, and myocardial fibrosis are important pathological changes of DCM. $\mathrm{H}_{2} \mathrm{~S}$ can improve LV function and inhibit myocardial hypertrophy and myocardial fibrosis to ameliorate DCM by suppressing inflammation, oxidative stress, and apoptosis induced by HG. Mechanism studies show that $\mathrm{H}_{2} \mathrm{~S}$ suppresses HG-induced oxidative stress by activating the nuclear factor erythroid 2-related factor 2(Nrf2)/antioxidant response element (ARE) pathway, decreases HG-induced apoptosis through inhibiting the c-Jun $\mathrm{N}$-terminal kinase (JNK)/p38 MAPK pathways, and activating the phosphatidylinositol 3kinase (PI3K)/Akt pathway (Zhou et al., 2015). Another pathway, the AMPK/mTOR pathway, is also involved with the $\mathrm{H}_{2} \mathrm{~S}$ protection of $\mathrm{H} 9 \mathrm{c} 2$ cells against HG-induced apoptosis. GYY4137 (a donor of $\mathrm{H}_{2} \mathrm{~S}$ ) treatment improves HG-induced cell viability decrement. Moreover, both GYY4137 and AICAR (an AMPK activator) increase AMPK phosphorylation, decrease mammalian target of rapamycin (mTOR) phosphorylation, and ameliorate HG-induced cell viability decrement. Moreover, AraA (an AMPK inhibitor) attenuates the cardioprotection of GYY4137. Collectively, it can be inferred that exogenous $\mathrm{H}_{2} \mathrm{~S}$ improves DCM through suppressing HG-induced apoptosis by activation of the AMPK/mTOR signal pathway (Wei et al., 2014).

Forkhead box protein $\mathrm{O} 1$ (FoxO1), which is a member of the FoxO family, plays a significant role in DCM through affecting oxidative stress, metabolism, inflammation, and apoptosis (Chistiakov et al., 2017). Exogenous $\mathrm{H}_{2} \mathrm{~S}$ can ameliorate cardiac 
function, myocardial fibrosis, and hypertrophy in diabetic mice by inhibiting oxidative stress and apoptosis induced by $\mathrm{HG}$. Mechanism studies show that $\mathrm{H}_{2} \mathrm{~S}$ promotes the phosphorylation level of FoxO1 and suppresses FoxO1 nuclear translocation in cardiomyocytes via S-sulfhydration, which inhibits apoptosis and suppresses oxidative stress by increasing the expression of antioxidant enzymes. Therefore, it can be inferred that exogenous $\mathrm{H}_{2} \mathrm{~S}$ ameliorates DCM via the FoxO1 pathway (Ye et al., 2018). FoxO1 may be an important target for $\mathrm{H}_{2} \mathrm{~S}$ to improve DCM. The Wnt/ $\beta$-catenin pathway also plays an important role in $\mathrm{H}_{2} \mathrm{~S}$ protection of DCM. Zhang and Ye found that $\mathrm{H}_{2} \mathrm{~S}$ could reduce the levels of ROS and malondialdehyde and increase the activities of superoxide dismutase, catalase, and glutathione peroxidase to attenuate HG-induced cardiomyocyte apoptosis and oxidative stress by suppressing the $\mathrm{Wnt} / \beta$-catenin pathway, which need to be further studied, especially the relationship between the Wnt/ $\beta$-catenin pathway and apoptosis (Zhang and Ye, 2019).

AGEs are proteins or lipids that become glycosylated after exposure to sugars. The receptor of advanced glycated product (RAGE) is a transmembrane receptor of the immunoglobulin superfamily, which can lead to oxidative stress, inflammation, and apoptosis. It has been found that $\mathrm{H}_{2} \mathrm{~S}$ can inhibit oxidative stress, inflammation, and apoptosis induced by RAGE overactivation through inhibiting RAGE dimer formation and impairing its protein stability (Zhou et al., 2017). Whether RAGE mediates the protective effect of $\mathrm{H}_{2} \mathrm{~S}$ on the diabetic myocardium is worth discussing. The signal transduction pathways involved in the above processes are summarized in Table $\mathbf{1}$.

\section{Exogenous $\mathrm{H}_{2} \mathrm{~S}$ Improves DCM by Regulating ERS}

It has been reported that ERS can be induced in the diabetic heart and participate in the pathogenesis of DCM (Lian et al., 2017). Cardiac lipid toxicity refers to the direct toxic effect of excessive

TABLE 1 | The signaling pathways involved in exogenous hydrogen sulfide $\left(\mathrm{H}_{2} \mathrm{~S}\right)$ improvements of diabetic cardiomyopathy by inhibiting oxidative stress, inflammation, and apoptosis.

\begin{tabular}{|c|c|c|}
\hline Signaling pathway & $\begin{array}{l}\text { The mode of } \\
\text { action of } \mathrm{H}_{2} \mathrm{~S} \text { on } \\
\text { signal pathway }\end{array}$ & Effects \\
\hline NF-кB pathway & Inhibition & Suppressing oxidative stress \\
\hline TLR4/ NF-кB pathway & Inhibition & $\begin{array}{l}\text { Suppressing inflammation and } \\
\text { apoptosis }\end{array}$ \\
\hline Nrf2/ARE pathway & Activation & Suppressing oxidative stress \\
\hline NK/p38 MAPK pathway & Inhibition & Suppressing apoptosis \\
\hline PI3K/Akt pathway & Activation & Suppressing apoptosis \\
\hline AMPK/mTOR pathway & Activation & Suppressing apoptosis \\
\hline FoxO1 pathway & Phosphorylation & $\begin{array}{l}\text { Suppressing oxidative stress } \\
\text { and apoptosis }\end{array}$ \\
\hline Wnt/ B-catenin pathway & Inhibition & $\begin{array}{l}\text { Suppressing oxidative stress } \\
\text { and apoptosis }\end{array}$ \\
\hline
\end{tabular}

NF- $\kappa B$, nuclear factor kappa-B; TLR4, Toll-like receptor 4; Nrf2, nuclear factor erythroid 2-related factor 2; ARE, antioxidant response element; JNK, c-Jun N-terminal kinase; p38 MAPK, p38 mitogen activated protein kinase; PI3K, phosphatidylinositol 3-kinase; mTOR, mammalian target of rapamycin; FoxO1, Forkhead box protein 01. lipid deposition on myocardial cell function, which may be an important part of the phenotype of DCM. Both exogenous $\mathrm{H}_{2} \mathrm{~S}$ and ERS inhibitors (4-PBA) can improve DCM by decreasing palmitic acid (PA)-induced myocardial injury. Similar results can be obtained in diabetic rats by using NaHS or 4-PBA. In addition, exogenous $\mathrm{H}_{2} \mathrm{~S}$ inhibits ERS in diabetic rats or PAinduced AC 16 cardiac cells by decreasing the expressions of CHOP, GRP78, and caspase-12; therefore, it can be inferred that exogenous $\mathrm{H}_{2} \mathrm{~S}$ can ameliorate DCM by inhibiting ERS (Guo et al., 2017). The relationship between ERS and apoptosis needs to be clarified, and how $\mathrm{H}_{2} \mathrm{~S}$ regulates lipid metabolism through ERS deserves further study. It has been reported that ROS overproduction in DCM is often accompanied by ERS and that ROS/ERS-mediated apoptosis is involved in the pathogenesis and development of DCM. Exogenous $\mathrm{H}_{2} \mathrm{~S}$ can improve the myocardial injury of diabetic rats, reduce the expression of ERSrelated proteins, and improve the injury of $\mathrm{H} 9 \mathrm{c} 2$ cells induced by HG. In HG-induced $\mathrm{H} 9 \mathrm{C} 2$ cells, exogenous $\mathrm{H}_{2} \mathrm{~S}$ notably reduced intracellular ROS levels, while an ROS scavenger decreases HGinduced apoptosis, indicating that ROS-induced cardiomyocyte apoptosis mediates the protective effect of exogenous $\mathrm{H}_{2} \mathrm{~S}$ on the diabetic myocardium. HG can induce ROS and ERS, while an ROS scavenger can inhibit the expression of ERSrelated proteins induced by $\mathrm{HG}$, suggesting that $\mathrm{HG}$ increases ROS level to promote ERS. In addition, in an HG-induced H9C2 cell, HG induces Mfn-2 expression, and siRNA targeting Mfn-2 reduces ROS-induced apoptosis and HG-induced ERS, indicating that HG increases ROS/ERS-mediated apoptosis via Mfn-2. Based on the above, it can be inferred that exogenous $\mathrm{H}_{2} \mathrm{~S}$ ameliorates DCM by decreasing ROS/ERS-mediated apoptosis through suppression of Mfn-2 expression (Yang et al., 2017). The relationship of ERS and Mfn-2 requires further study.

\section{Exogenous $\mathrm{H}_{2} \mathrm{~S}$ Improves DCM by Regulating Autophagy}

Type 2 diabetes is characterized by protein misfolding and aggregation, which results in mitochondrial damage, ROS production, and apoptosis and leads to ubiquitin aggregates (Chiti and Dobson, 2006; Shang and Taylor, 2011; Sato et al., 2014). Ubiquitin aggregation, which also leads to apoptosis and ROS production, is mainly eliminated by autophagy (Zhang Y. et al., 2017; Grumati and Dikic, 2018). Autophagy plays a protective role in DCM by clearing misfolded protein and ubiquitin (Pei et al., 2018). In diabetic mice, exogenous $\mathrm{H}_{2} \mathrm{~S}$ can ameliorate DCM by decreasing ROS production. Exogenous $\mathrm{H}_{2} \mathrm{~S}$ also can promote the degradation of autophagosome content, decrease the expression of p62, and increase the expression of microtubule associated protein 1 light chain 3 II (LC3II), autophagy associated protein 7 (Atg7), and Beclin1, indicating that exogenous $\mathrm{H}_{2} \mathrm{~S}$ promotes autophagy. Moreover, exogenous $\mathrm{H}_{2} \mathrm{~S}$ increases the expression of kelch-like ECH related protein 1 (keap-1) by decreasing its ubiquitylation, and Keap-1 siRNA inhibits the effect of exogenous $\mathrm{H}_{2} \mathrm{~S}$ on autophagy in the cardiomyocyte of diabetic rats, indicating that exogenous $\mathrm{H}_{2} \mathrm{~S}$ promotes autophagy through keap-1. Further research shows that 1,4-dithiothreitol, an inhibitor of disulfide bonds, counteracts the 
effects of $\mathrm{H}_{2} \mathrm{~S}$ on keap-1, ubiquitin aggregates clearance and ROS production in HG-induced $\mathrm{H} 9 \mathrm{C} 2$ cells, and $\mathrm{H}_{2} \mathrm{~S}$ can promote the formation of disulfide between two keap-1 molecules, which indicates that exogenous $\mathrm{H}_{2} \mathrm{~S}$ suppresses Keap-1 ubiquitylation through promoting its disulfide formation. From the above results, it can be inferred that exogenous $\mathrm{H}_{2} \mathrm{~S}$ improves DCM by promoting ubiquitin aggregation clearance through promoting autophagy via ubiquitylation of Keap-1, which contributes to ROS scavenging and provides a new mechanism for the antioxidation of $\mathrm{H}_{2} \mathrm{~S}$. In addition, exogenous $\mathrm{H}_{2} \mathrm{~S}$ has no notable effect on Nrf2 nuclear translocation, indicating that its antioxidant effect is not related with the keap-1/Nrf2 signaling pathway (Wu et al., 2017). The mechanism of exogenous $\mathrm{H}_{2} \mathrm{~S}$ acting as an antioxidant through autophagy needs further study.

\section{Exogenous $\mathrm{H}_{2} \mathrm{~S}$ Improves DCM by Improving Cardiac Mitochondrial Function}

Recent evidence suggests that DCM is associated with metabolic abnormalities, more often with mitochondrial dysfunction. Sirtuin 3 (SIRT3) belongs to the nicotinamide adenine dinucleotide (NAD)-dependent deacetylase family and is

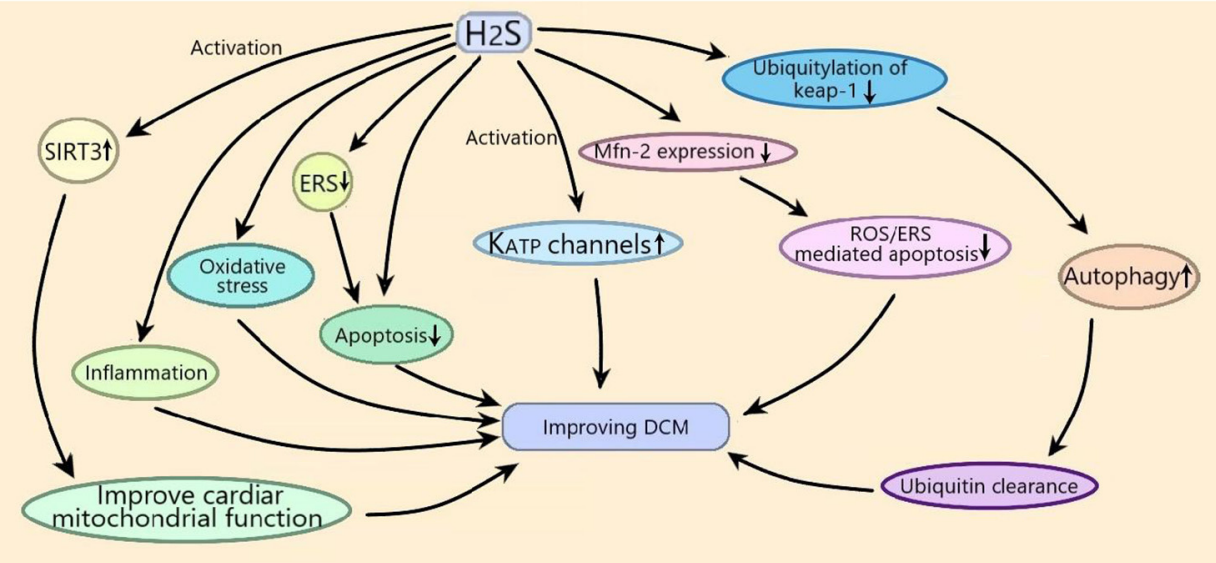

FIGURE 2 | Schematic diagram of the mechanism of exogenous $\mathrm{H}_{2} \mathrm{~S}$ improving diabetic cardiomyopathy (DCM). Exogenous $\mathrm{H}_{2} \mathrm{~S}$ improves DCM by suppressing oxidative stress, inflammation, and apoptosis. Exogenous $\mathrm{H}_{2} \mathrm{~S}$ improves DCM by inhibiting apoptosis through suppressing endoplasmic reticulum stress (ERS) or inhibiting reactive oxygen species (ROS)/ERS-mediated apoptosis through suppressing Mfn-2 expression. Exogenous $\mathrm{H}_{2} \mathrm{~S}$ improves DCM by suppressing ubiquitylation of kelch-like ECH related protein 1 (Keap-1) to promote autophagy for ubiquitin clearance. Exogenous $\mathrm{H}_{2} \mathrm{~S}$ improves DCM by improving cardiac mitochondrial function through activating sirtuin 3 (SIRT3). Exogenous $\mathrm{H}_{2} \mathrm{~S}$ could improve DCM by activating $\mathrm{K}_{\text {ATP }}$ channels.

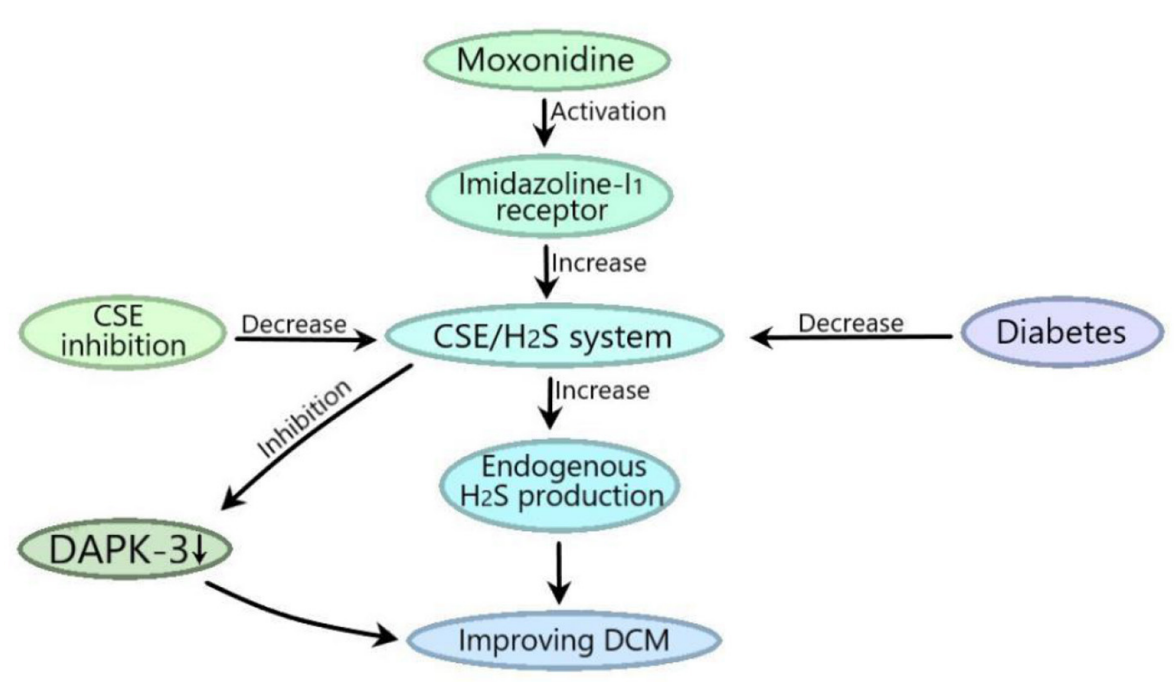

FIGURE 3 | CSE-derived $\mathrm{H}_{2} \mathrm{~S}$ mediates the moxonidine improvements of DCM. Diabetic state can inhibit the CSE/ $\mathrm{H}_{2} \mathrm{~S}$ system, and moxonidine can activate myocardial imidazoline 11 receptor to improve DCM by promoting the CSE $/ \mathrm{H}_{2} \mathrm{~S}$ system to increase endogenous $\mathrm{H}_{2} \mathrm{~S}$ production or by promoting the CSE/ $\mathrm{H}_{2} \mathrm{~S}$ system to inhibit death related protein kinase 3 (DAPK-3). 
the major mitochondrial deacetylase of lysine residues. In cardiac mitochondria of diabetic mice, cardiac mitochondrial respiratory capacities, ATP synthesis, and the expression and activity of SIRT3 were decreased, and exogenous $\mathrm{H}_{2} \mathrm{~S}$ increased the expression and activity of SIRT3 by restoring the ratio of $\mathrm{NAD}^{+} / \mathrm{NADH}$, and decreased the acetylation levels of the mitochondrial respiratory complex enzymes to improve cardiac mitochondrial dysfunction. SiRNA-mediated SIRT3 silencing increases the acetylation level of mitochondrial respiratory complexes, while exogenous $\mathrm{H}_{2} \mathrm{~S}$ partially restores the acetylation level of these enzymes. Collectively, it can be inferred that exogenous $\mathrm{H}_{2} \mathrm{~S}$ improves DCM by improving cardiac mitochondrial function through increasing the expression of SIRT3 to regulate the lysine acetylation of mitochondrial respiratory complexes (Sun Y. et al., 2019). The role of SIRT3 in $\mathrm{H}_{2} \mathrm{~S}$ improving DCM remains to be further studied.

\section{Exogenous $\mathrm{H}_{2} \mathrm{~S}$ Improves DCM by Activating $\mathrm{K}_{\text {ATP }}$ Channels}

The $\mathrm{K}_{\mathrm{ATP}}$ channels are abundant in the myocardium (Nichols and Lederer, 1991). The opening of $K_{\text {ATP }}$ channels can reduce the apoptosis induced by oxidative stress in cardiac cells to improve DCM (Akao et al., 2001). Liang et al. found that the expression levels of $\mathrm{K}_{\mathrm{ATP}}$ channels were decreased by $\mathrm{HG}$, which was abolished by exogenous $\mathrm{H}_{2} \mathrm{~S}$. HG or $\mathrm{K}_{\text {ATP }}$ channel blocker could induce in $\mathrm{H} 9 \mathrm{c} 2$ cardiac cells considerable injuries, including reducing cell viability, increasing apoptosis, ROS generation, and cleaved caspase- 3 expression, as well as the loss of MMP. However, exogenous $\mathrm{H}_{2} \mathrm{~S}$ or $\mathrm{K}_{\text {ATP }}$ channel openers could reverse the changes. Collectively, it could be inferred that exogenous $\mathrm{H}_{2} \mathrm{~S}$ could improve DCM by activating $\mathrm{K}_{\mathrm{ATP}}$ channels. Moreover, an ROS scavenger could ameliorate the reduction in the expression levels of the $\mathrm{K}_{\mathrm{ATP}}$ channel induced by $\mathrm{HG}$, and $\mathrm{K}_{\mathrm{ATP}}$ channel openers could decrease $\mathrm{HG}$-induced ROS production, indicating that there is an interaction between $\mathrm{K}_{\mathrm{ATP}}$ channels and ROS and that the interaction is involved in the above $\mathrm{H}_{2} \mathrm{~S}$ improvement of DCM (Liang et al., 2016). The role of $\mathrm{K}_{\mathrm{ATP}}$ channels in the improvement of DCM by $\mathrm{H}_{2} \mathrm{~S}$, especially the interaction between $\mathrm{K}_{\mathrm{ATP}}$ channels and ROS, needs to be further studied. $\mathrm{K}_{\text {ATP }}$ channels will become an important target for $\mathrm{H}_{2} \mathrm{~S}$ to improve DCM.

\section{The Roles of Endogenous $\mathrm{H}_{2} \mathrm{~S}$ in DCM}

In addition to exogenous $\mathrm{H}_{2} \mathrm{~S}$, endogenous $\mathrm{H}_{2} \mathrm{~S}$ can also improve DCM. El-Sayed et al. found that HG decreased CSE expression/activity, $\mathrm{H}_{2} \mathrm{~S}$, and serum adiponectin level and increased myocardial imidazoline $I_{1}$ receptor expression, while moxonidine (imidazoline $\mathrm{I}_{1}$ receptor agonist) abolished the above effects of $\mathrm{HG}$ and improved the glycemic state; reversed myocardial hypertrophy, hypertension, and baroreflex dysfunction in streptozocin (STZ)-treated rats; and inhibited the expression of death associated protein kinase-3 (DAPK3) to play cardiovascular protective effects in diabetic mice. Moreover, inhibition of CSE decreased endogenous $\mathrm{H}_{2} \mathrm{~S}$ production and counteracted moxonidine protective effects, indicating that CSE-derived $\mathrm{H}_{2} \mathrm{~S}$ might mediate the cardiovascular protective effects of moxonidine in diabetes
(El-Sayed et al., 2016). So far, there are few studies on moxonidine and endogenous $\mathrm{H}_{2} \mathrm{~S}$, so how moxonidine promotes the production of CSE-derived $\mathrm{H}_{2} \mathrm{~S}$ remains to be further explored.

\section{CONCLUSION}

Exogenous $\mathrm{H}_{2} \mathrm{~S}$ can improve DCM by suppressing oxidative stress, inflammation, and apoptosis. Exogenous $\mathrm{H}_{2} \mathrm{~S}$ improves DCM by inhibiting apoptosis through suppressing ERS or inhibiting ROS/ERS-mediated apoptosis through suppressing Mfn-2 expression. Exogenous $\mathrm{H}_{2} \mathrm{~S}$ improves DCM by suppressing ubiquitylation of Keap-1 to promote autophagy for ubiquitin clearance. Exogenous $\mathrm{H}_{2} \mathrm{~S}$ improves DCM by improving cardiac mitochondrial function through activating SIRT3. Exogenous $\mathrm{H}_{2} \mathrm{~S}$ could improve DCM by activating $\mathrm{K}_{\text {ATP }}$ channels (Figure 2). It can be seen from the above that the anti-inflammatory, anti-apoptotic, and antioxidant effects of $\mathrm{H}_{2} \mathrm{~S}$ have potential therapeutic value in DCM, but its mechanism has not been fully studied, especially the signal transduction pathways involved. Autophagy, the NLRP3 inflammasome, and ERS are the regulated targets of $\mathrm{H}_{2} \mathrm{~S}$ and are involved in the process of improving DCM by $\mathrm{H}_{2} \mathrm{~S}$, so the interaction among the three in $\mathrm{H}_{2} \mathrm{~S}$ improvement of DCM should be studied in the future. ERS and NLRP3 inflammasome crosstalk has been reported to play an important role in metabolic disorders (Ji et al., 2019), and $\mathrm{H}_{2} \mathrm{~S}$ regulates the ERS/NLRP3 inflammasome in many diseases (Wang et al., 2020a,b), so whether $\mathrm{H}_{2} \mathrm{~S}$ can regulate the ERS/NLRP3 inflammasome to improve diabetes deserves to be studied. In addition, the existing $\mathrm{H}_{2} \mathrm{~S}$ releasers cannot fully meet the requirements of research and development of $\mathrm{H}_{2} \mathrm{~S}$ related drugs, so the development of new $\mathrm{H}_{2} \mathrm{~S}$ releasers is very important for the application of $\mathrm{H}_{2} \mathrm{~S}$ related drugs in the treatment of clinical diseases.

Diabetic states can inhibit endogenous $\mathrm{H}_{2} \mathrm{~S}$-producing enzyme CSE, while the activation of myocardial imidazoline I1 receptor with moxonidine can improve DCM by increasing endogenous $\mathrm{H}_{2} \mathrm{~S}$ via CSE or increase CSE expression to inhibit DAPK-3 to ameliorate DCM (Figure 3). Myocardial imidazoline I1 receptor is an important target for the development of therapeutic drugs for DCM.

In conclusion, with further research, $\mathrm{H}_{2} \mathrm{~S}$ could provide new ways of treating DCM.

\section{AUTHOR CONTRIBUTIONS}

HW: devised, writing, and funded with this review. XiaL: drawing. SZ: writing and funded with this review. XW: writing. All authors contributed to the article and approved the submitted version.

\section{FUNDING}

This work was supported by grants from key scientific and technological projects in Henan Province, China (Grant No. 202102310153), the National Natural Science Foundation of 
China (Grant No. 81903444), the China Postdoctoral Science Foundation (Grant No. 2018M640674), and the Key Science and Technology Program of Henan Province in China (Grant No. 192102310145).

\section{REFERENCES}

Acar, E., Ural, D., Bildirici, U., Sahin, T., and Yilmaz, I. (2011). Diabetic cardiomyopathy. Anatolian J. Cardiol. 11, 732-737. doi: 10.5152/akd.2011.196

Akao, M., Ohler, A., O'rourke, B., and Marban, E. (2001). Mitochondrial ATPsensitive potassium channels inhibit apoptosis induced by oxidative stress in cardiac cells. Circ. Res. 88, 1267-1275. doi: 10.1161/hh1201.092094

Al Hroob, A. M., Abukhalil, M. H., Hussein, O. E., and Mahmoud, A. M. (2019). Pathophysiological mechanisms of diabetic cardiomyopathy and the therapeutic potential of epigallocatechin-3-gallate. Biomed. Pharmacotherapy 109, 2155-2172. doi: 10.1016/j.biopha.2018.11.086

Al-Rasheed, N. M., Al-Rasheed, N. M., Hasan, I. H., Al-Amin, M. A., AlAjmi, H. N., Mohamad, R. A., et al. (2017). Simvastatin ameliorates diabetic cardiomyopathy by attenuating oxidative stress and inflammation in rats. Oxid. Med. Cell. Longev. 2017, 1092015. doi: 10.1155/2017/1092015

Balakumar, P., Maung,-U., K., and Jagadeesh, G. (2016). Prevalence and prevention of cardiovascular disease and diabetes mellitus. Pharmacol. Res. 113, 600-609. doi: 10.1016/j.phrs.2016.09.040

Behera, J., Tyagi, S. C., and Tyagi, N. (2019). Role of hydrogen sulfide in the musculoskeletal system. Bone 124, 33-39. doi: 10.1016/j.bone.2019.03.034

Boudina, S., and Abel, E. D. (2007). Diabetic cardiomyopathy revisited. Circulation 115, 3213-3223. doi: 10.1161/CIRCULATIONAHA.106.679597

Chen, S. L., Khan, Z. A., Cukiernik, M., and Chakrabarti, S. (2003). Differential activation of NF- $\kappa$ B and AP-1 in increased fibronectin synthesis in target organs of diabetic complications. Am. J. Physiol. Endocrinol. Metab. 284, E1089-E1097. doi: 10.1152/ajpendo.00540.2002

Chistiakov, D. A., Orekhov, A. N., and Bobryshev, Y. V. (2017). The impact of FOXO-1 to cardiac pathology in diabetes mellitus and diabetes-related metabolic abnormalities. Int. J. Cardiol. 245, 236-244. doi: 10.1016/j.ijcard.2017.07.096

Chiti, F., and Dobson, C. M. (2006). Protein misfolding, functional amyloid, and human disease. Annu. Rev. Biochem. 75, 333-366. doi: 10.1146/annurev.biochem.75.101304.123901

Citi, V., Piragine, E., Testai, L., Breschi, M. C., Calderone, V., and Martelli, A. (2018). The role of hydrogen sulfide and $\mathrm{H}_{2} \mathrm{~S}$-donors in myocardial protection against ischemia/reperfusion injury. Curr. Med. Chem. 25, 4380-4401. doi: 10.2174/0929867325666180212120504

Darville, M. I., and Eizirik, D. L. (2001). Cytokine induction of Fas gene expression in insulin-producing cells requires the transcription factors NF- $\mathrm{BB}$ and C/EBP. Diabetes 50, 1741-1748. doi: 10.2337/diabetes.50.8.1741

Dillmann, W. H. (2019). Diabetic cardiomyopathy what is it and can it be fixed? Circ. Res. 124, 1160-1162. doi: 10.1161/CIRCRESAHA.118.314665

El-Sayed, S. S., Zakaria, M. N. M., Abdel-Ghany, R. H., and Abdel-Rahman, A. A. (2016). Cystathionine-gamma lyase-derived hydrogen sulfide mediates the cardiovascular protective effects of moxonidine in diabetic rats. Eur. J. Pharmacol. 783, 73-84. doi: 10.1016/j.ejphar.2016.04.054

El-Seweidy, M. M., Sadik, N. A. H., and Shaker, O. G. (2011). Role of sulfurous mineral water and sodium hydrosulfide as potent inhibitors of fibrosis in the heart of diabetic rats. Arch. Biochem. Biophys. 506, 48-57. doi: 10.1016/j.abb.2010.10.014

Felicio, J. S., Koury, C. C., Carvalho, C. T., Abrahao Neto, J. F., Mileo, K. B., Arbage, T. P., et al. (2016). Present insights on cardiomyopathy in diabetic patients. Curr. Diabetes Rev. 12, 384-395. doi: 10.2174/1573399812666150914120529

Greaney, J. L., Kutz, J. L., Shank, S. W., Jandu, S., Santhanam, L., and Alexander, L. M. (2017). Impaired hydrogen sulfide-mediated vasodilation contributes to microvascular endothelial dysfunction in hypertensive adults. Hypertension 69, 902. doi: 10.1161/HYPERTENSIONAHA.116.08964

Grumati, P., and Dikic, I. (2018). Ubiquitin signaling and autophagy. J. Biol. Chem. 293, 5404-5413. doi: 10.1074/jbc.TM117.000117

Guo, F.-F., Yu, T.-C., Hong, J., and Fang, J.-Y. (2016). Emerging roles of hydrogen sulfide in inflammatory and neoplastic colonic diseases. Front. Physiol. 7:156. doi: 10.3389/fphys.2016.00156
Guo, R., Wu, Z., Jiang, J., Liu, C., Wu, B., Li, X., et al. (2017). New mechanism of lipotoxicity in diabetic cardiomyopathy: deficiency of endogenous $\mathrm{H}_{2} \mathrm{~S}$ production and ER stress. Mech. Ageing Dev. 162, 46-52. doi: 10.1016/j.mad.2016.11.005

Han, M., Liu, D., Qiu, J., Yuan, H., Hu, Q., Xue, H., et al. (2020). Evaluation of $\mathrm{H}_{2} \mathrm{~S}$-producing enzymes in cerebrospinal fluid and its relationship with interleukin-6 and neurologic deficits in subarachnoid hemorrhage. Biomed. Pharmacotherapy 123, 109722. doi: 10.1016/j.biopha.2019.109722

Hein, S., Arnon, E., Kostin, S., Schonburg, M., Elsasser, A., Polyakova, V., et al. (2003). Progression from compensated hypertrophy to failure in the pressure-overloaded human heart - Structural deterioration and compensatory mechanisms. Circulation 107, 984-991. doi: 10.1161/01.CIR.0000051865.66123.B7

Htay, T., Soe, K., Lopez-Perez, A., Amy Hoanganh, D., Romagosa, M. A., and Aung, K. (2019). Mortality and cardiovascular disease in type 1 and type 2 diabetes. Curr. Cardiol. Rep. 21. doi: 10.1007/s11886-019-1133-9

Huang, Z., Zhuang, X., Xie, C., Hu, X., Dong, X., Guo, Y., et al. (2016). Exogenous hydrogen sulfide attenuates high glucose-induced cardiotoxicity by inhibiting NLRP3 inflammasome activation by suppressing TLR4/NF- $\kappa \mathrm{B}$ pathway in H9c2 cells. Cell. Physiol. Biochem. 40, 1578-1590. doi: 10.1159/000453208

Ji, T., Han, Y., Yang, W., Xu, B., Sun, M., Jiang, S., et al. (2019). Endoplasmic reticulum stress and NLRP3 inflammasome: crosstalk in cardiovascular and metabolic disorders. J. Cell. Physiol. 234, 14773-14782. doi: 10.1002/jcp.28275

Jia, G., Hill, M. A., and Sowers, J. R. (2018a). Diabetic cardiomyopathy: an update of mechanisms contributing to this clinical entity. Circ. Res. 122, 624-638. doi: 10.1161/CIRCRESAHA.117.311586

Jia, G., Whaley-Connell, A., and Sowers, J. R. (2018b). Diabetic cardiomyopathy: a hyperglycaemia- and insulin-resistance-induced heart disease. Diabetologia 61, 21-28. doi: 10.1007/s00125-017-4390-4

Jin, S., Teng, X., Xiao, L., Xue, H., Guo, Q., Duan, X., et al. (2017). Hydrogen sulfide ameliorated L-NAME-induced hypertensive heart disease by the Akt/eNOS/NO pathway. Exp. Biol. Med. 242, 1831-1841. doi: $10.1177 / 1535370217732325$

Kar, S., Kambis, T. N., and Mishra, P. K. (2019). Hydrogen sulfide-mediated regulation of cell death signaling ameliorates adverse cardiac remodeling and diabetic cardiomyopathy. Am. J. Physiol. Heart Circ. Physiol. 316, H1237H1252. doi: 10.1152/ajpheart.00004.2019

Karbasforooshan, H., and Karimi, G. (2017). The role of SIRT1 in diabetic cardiomyopathy. Biomed. Pharmacotherapy 90, 386-392. doi: 10.1016/j.biopha.2017.03.056

Karmin, O., and Siow, Y. L. (2018). Metabolic imbalance of homocysteine and hydrogen sulfide in kidney disease. Curr. Med. Chem. 25, 367-377. doi: $10.2174 / 0929867324666170509145240$

Li, J., Teng, X., Jin, S., Dong, J., Guo, Q., Tian, D., et al. (2019a). Hydrogen sulfide improves endothelial dysfunction by inhibiting the vicious cycle of NLRP3 inflammasome and oxidative stress in spontaneously hypertensive rats. J. Hypertens. 37, 1633-1643. doi: 10.1097/HJH.0000000000002101

Li, J., Yuan, Y.-Q., Zhang, L., Zhang, H., Zhang, S.-W., Zhang, Y., et al. (2019b). Exogenous hydrogen sulfide protects against high glucose-induced apoptosis and oxidative stress by inhibiting the STAT3/HIF-1 alpha pathway in H9c2 cardiomyocytes. Exp. Therap. Med. 18, 3948-3958. doi: 10.3892/etm.2019.8036

Lian, J., Chen, J., Yuan, Y., Chen, J., Sayed, M. D. M., Luo, L., et al. (2017). Cortex Mori Radicis extract attenuates myocardial damages in diabetic rats by regulating ERS. Biomed. Pharmacotherapy 90, 777-785. doi: 10.1016/j.biopha.2017.03.097

Liang, W., Chen, J., Mo, L., Ke, X., Zhang, W., Zheng, D., et al. (2016). ATP-sensitive $\mathrm{K}+$ channels contribute to the protective effects of exogenous hydrogen sulfide against high glucose-induced injury in $\mathrm{H} 9 \mathrm{c} 2$ cardiac cells. Int. J. Mol. Med. 37, 763-772. doi: 10.3892/ijmm.2016.2467

Mahmoud, A. M. (2017). Exercise amaliorates metabolic disturbances and oxidative stress in diabetic cardiomyopathy: possible underlying mecchanisms. Adv. Exp. Med. Biol. 999, 207-230. doi: 10.1007/978-981-10-4307-9_12 
Mard, S. A., Ahmadi, I., Ahangarpour, A., Gharib-Naseri, M. K., and Badavi, M. (2016). Delayed gastric emptying in diabetic rats caused by decreased expression of cystathionine gamma lyase and $\mathrm{H}_{2} \mathrm{~S}$ synthesis: in vitro and in vivo studies. Neurogastroenterol. Motil. 28, 1677-1689. doi: 10.1111/nmo.12867

Nichols, C. G., and Lederer, W. J. (1991). Adenosine triphosphate-sensitive potassium channels in the cardiovascular system. Am. J. Physiol. 261, H1675-1686. doi: 10.1152/ajpheart.1991.261.6.H1675

Pei, Z., Deng, Q., Babcock, S. A., He, E. Y., Ren, J., and Zhang, Y. (2018). Inhibition of advanced glycation endproduct (AGE) rescues against streptozotocininduced diabetic cardiomyopathy: role of autophagy and ER stress. Toxicol. Lett. 284, 10-20. doi: 10.1016/j.toxlet.2017.11.018

Piragine, E., and Calderone, V. (2020). Pharmacological modulation of the hydrogen sulfide $\left(\mathrm{H}_{2} \mathrm{~S}\right)$ system by dietary $\mathrm{H}_{2} \mathrm{~S}$-donors: a novel promising strategy in the prevention and treatment of type 2 diabetes mellitus. Phytotherapy Res. doi: 10.1002/ptr.6923

Pol, A., Renkema, G. H., Tangerman, A., Winkel, E. G., Engelke, U. F., De Brouwer, A. P. M., et al. (2018). Mutations in SELENBP1, encoding a novel human methanethiol oxidase, cause extraoral halitosis. Nat. Genet. 50, 120-129. doi: 10.1038/s41588-017-0006-7

Poornima, I. G., Parikh, P., and Shannon, R. P. (2006). Diabetic cardiomyopathy - the search for a unifying hypothesis. Circ. Res. 98, 596-605. doi: 10.1161/01.RES.0000207406.94146.c2

Qian, L.-L., Liu, X.-Y., Chai, Q., and Wang, R.-X. (2018). Hydrogen sulfide in diabetic complications: focus on molecular mechanisms. Endocr. Metab. Immune Disord. Drug Targets 18, 470-476. doi: $10.2174 / 1871530318666180426100532$

Rose, P., Moore, P. K., and Zhu, Y. Z. (2017). $\mathrm{H}_{2} \mathrm{~S}$ biosynthesis and catabolism: new insights from molecular studies. Cell. Mol. Life Sci. 74, 1391-1412. doi: 10.1007/s00018-016-2406-8

Russo, I., and Frangogiannis, N. G. (2016). Diabetes-associated cardiac fibrosis: cellular effectors, molecular mechanisms and therapeutic opportunities. J. Mol. Cell. Cardiol. 90, 84-93. doi: 10.1016/j.yjmcc.2015.12.011

Sato, A., Asano, T., Isono, M., Ito, K., and Asano, T. (2014). Panobinostat synergizes with bortezomib to induce endoplasmic reticulum stress and ubiquitinated protein accumulation in renal cancer cells. BMC Urol. 14:71. doi: 10.1186/1471-2490-14-71

Shang, F., and Taylor, A. (2011). Ubiquitin-proteasome pathway and cellular responses to oxidative stress. Free Radic. Biol. Med. 51, 5-16. doi: 10.1016/j.freeradbiomed.2011.03.031

Sharma, A., Tate, M., Mathew, G., Vince, J. E., Ritchie, R. H., and De Haan, J. B. (2018). Oxidative stress and NLRP3-inflammasome activity as significant drivers of diabetic cardiovascular complications: therapeutic implications. Front. Physiol. 9:114. doi: 10.3389/fphys.2018.00114

Shen, F., Zhao, C.-S., Shen, M.-F., Wang, Z., and Chen, G. (2019). The role of hydrogen sulfide in gastric mucosal damage. Med. Gas Res. 9, 88-92. doi: $10.4103 / 2045-9912.260650$

Sulaiman, M., Matta, M. J., Sunderesan, N. R., Gupta, M. P., Periasamy, M., and Gupta, M. (2010). Resveratrol, an activator of SIRT1, upregulates sarcoplasmic calcium ATPase and improves cardiac function in diabetic cardiomyopathy. Am. J. Physiol. Heart Circ. Physiol. 298, H833-H843. doi: 10.1152/ajpheart.00418.2009

Sun, H.-J., Wu, Z.-Y., Cao, L., Zhu, M.-Y., Liu, T.-T., Guo, L., et al. (2019). Hydrogen sulfide: recent progression and perspectives for the treatment of diabetic nephropathy. Molecules 24, 2857. doi: 10.3390/molecules24152857

Sun, Y., Teng, Z., Sun, X., Zhang, L., Chen, J., Wang, B., et al. (2019). Exogenous $\mathrm{H}_{2} \mathrm{~S}$ reduces the acetylation levels of mitochondrial respiratory enzymes via regulating the $\mathrm{NAD}(+)$-SIRT3 pathway in cardiac tissues of $\mathrm{db} / \mathrm{db}$ mice. Am. J. Physiol. Endocrinol. Metab. 317, E284-E297. doi: 10.1152/ajpendo.00326.2018

Szabo, C. (2012). Roles of hydrogen sulfide in the pathogenesis of diabetes mellitus and its complications. Antioxid. Redox Signal. 17, 68-80. doi: $10.1089 /$ ars.2011.4451

Taniguchi, S., Kang, L., Kimura, T., and Niki, I. (2011). Hydrogen sulphide protects mouse pancreatic beta-cells from cell death induced by oxidative stress, but not by endoplasmic reticulum stress. Br. J. Pharmacol. 162, 1171-1178. doi: 10.1111/j.1476-5381.2010.01119.x

Tillquist, M. N., and Maddox, T. M. (2012). Update on diabetic cardiomyopathy: inches forward, miles to go. Curr. Diabetes Rep. 12, 305-313. doi: 10.1007/s11892-012-0274-7
Tocmo, R., and Parkin, K. (2019). S-1-propenylmercaptocysteine protects murine hepatocytes against oxidative stress via persulfidation of Keap1 and activation of Nrf2. Free Radic. Biol. Med. 143, 164-175. doi: 10.1016/j.freeradbiomed.2019.07.022

Varma, U., Koutsifeli, P., Benson, V. L., Mellor, K. M., and Delbridge, L. M. D. (2018). Molecular mechanisms of cardiac pathology in diabetes Experimental insights. Biochim. Biophys. Acta Mol. Basis Dis. 1864, 1949-1959. doi: 10.1016/j.bbadis.2017.10.035

Wang, H., Shi, X., Qiu, M., Lv, S., and Liu, H. (2020a). Hydrogen sulfide plays an important protective role through influencing endoplasmic reticulum stress in diseases. Int. J. Biol. Sci. 16, 264-271. doi: 10.7150/ijbs.38143

Wang, H., Shi, X., Qiu, M., Lv, S., Zheng, H., Niu, B., et al. (2020b). Hydrogen sulfide plays an important role by influencing NLRP3 inflammasome. Int. J. Biol. Sci. 16, 2752-2760. doi: 10.7150/ijbs.47595

Wang, H., Zhong, P., and Sun, L. (2019). Exogenous hydrogen sulfide mitigates NLRP3 inflammasome-mediated inflammation through promoting autophagy via the AMPK-mTOR pathway. Biol. Open 8, bio043653. doi: 10.1242/bio.043653

Wang, P., Chen, F., Wang, W., and Zhang, X.-D. (2019). Hydrogen sulfide attenuates high glucose-induced human retinal pigment epithelial cell inflammation by inhibiting ROS formation and NLRP3 inflammasome activation. Mediat. Inflamm. 2019, 8908960. doi: 10.1155/2019/8908960

Wei, W.-B., Hu, X., Zhuang, X.-D., Liao, L.-Z., and Li, W.-D. (2014). GYY4137, a novel hydrogen sulfide-releasing molecule, likely protects against high glucoseinduced cytotoxicity by activation of the AMPK/mTOR signal pathway in H9c2 cells. Mol. Cell. Biochem. 389, 249-256. doi: 10.1007/s11010-013-1946-6

Wu, J., Tian, Z., Sun, Y., Lu, C., Liu, N., Gao, Z., et al. (2017). Exogenous $\mathrm{H}_{2} \mathrm{~S}$ facilitating ubiquitin aggregates clearance via autophagy attenuates type 2 diabetes-induced cardiomyopathy. Cell Death Dis. 8, e2992. doi: $10.1038 /$ cddis. 2017.380

Xiao, Q., Ying, J., Xiang, L., and Zhang, C. (2018). The biologic effect of hydrogen sulfide and its function in various diseases. Medicine 97, e13065. doi: 10.1097/MD.0000000000013065

Yamagishi, S., Edelstein, D., Du, X. L., and Brownlee, M. (2001). Hyperglycemia potentiates collagen-induced platelet activation through mitochondrial superoxide overproduction. Diabetes 50, 1491-1494. doi: $10.2337 /$ diabetes.50.6.1491

Yang, F., Yu, X., Li, T., Wu, J., Zhao, Y., Liu, J., et al. (2017). Exogenous $\mathrm{H}_{2} \mathrm{~S}$ regulates endoplasmic reticulum-mitochondria cross-talk to inhibit apoptotic pathways in STZ-induced type I diabetes. Am. J. Physiol. Endocrinol. Metab. 312, E190-E203. doi: 10.1152/ajpendo.00196.2016

Yang, G., Yang, W., Wu, L., and Wang, R. (2007). $\mathrm{H}_{2} \mathrm{~S}$, endoplasmic reticulum stress, and apoptosis of insulin-secreting beta cells. J. Biol. Chem. 282, 16567-16576. doi: 10.1074/jbc.M700605200

Yang, J., Minkler, P., Grove, D., Wang, R., Willard, B., Dweik, R., et al. (2019). Nonenzymatic hydrogen sulfide production from cysteine in blood is catalyzed by iron and vitamin B-6. Commun. Biol. 2, 19. doi: 10.1038/s42003-019-0431-5

Yao, Q., Ke, Z.-Q., Guo, S., Yang, X.-S., Zhang, F.-X., Liu, X.-F., et al. (2018). Curcumin protects against diabetic cardiomyopathy by promoting autophagy and alleviating apoptosis. J. Mol. Cell. Cardiol. 124, 26-34. doi: 10.1016/j.yjmcc.2018.10.004

Ye, P., Gu, Y., Zhu, Y.-R., Chao, Y.-L., Kong, X.-Q., Luo, J., et al. (2018). Exogenous hydrogen sulfide attenuates the development of diabetic cardiomyopathy via the FoxO1 pathway. J. Cell. Physiol. 233, 9786-9798. doi: 10.1002/jcp.26946

Ye, Y., Bajaj, M., Yang, H.-C., Perez-Polo, J. R., and Birnbaum, Y. (2017). SGLT-2 inhibition with dapagliflozin reduces the activation of the Nlrp3/ASC inflammasome and attenuates the development of diabetic cardiomyopathy in mice with type 2 diabetes. Further augmentation of the effects with saxagliptin, a DPP4 inhibitor. Cardiovasc. Drugs Therapy 31, 119-132. doi: 10.1007/s10557-017-6725-2

Zhang, D., Du, J., Tang, C., Huang, Y., and Jin, H. (2017). $\mathrm{H}_{2} \mathrm{~S}$-induced sulfhydration: biological function and detection methodology. Front. Pharmacol. 8:608. doi: 10.3389/fphar.2017.00608

Zhang, M., and Ye, M. (2019). Hydrogen sulfide attenuates high glucose-induced myocardial injury in rat cardiomyocytes by suppressing Wnt/beta-catenin pathway. Curr. Med. Sci. 39, 938-946. doi: 10.1007/s11596-019-2120-5

Zhang, Y., Chen, X., Zhao, Y., Ponnusamy, M., and Liu, Y. (2017). The role of ubiquitin proteasomal system and autophagy-lysosome pathway in 
Alzheimer's disease. Rev. Neurosci. 28, 861-868. doi: 10.1515/revneuro-201 7-0013

Zhao, A.-S., Zou, D., Wang, H.-H., Han, X., Yang, P., and Huang, N. (2019). Hydrogen sulphide-releasing aspirin enhances cell capabilities of anti-oxidative lesions and anti-inflammation. Med. Gas Res. 9, 145-152. doi: 10.4103/2045-9912.266990

Zhao, M.-X., Zhou, B., Ling, L., Xiong, X.-Q., Zhang, F., Chen, Q., et al. (2017). Salusin-beta contributes to oxidative stress and inflammation in diabetic cardiomyopathy. Cell Death Dis. 8, e2690. doi: 10.1038/cddis.2017.106

Zhou, H., Ding, L., Wu, Z., Cao, X., Zhang, Q., Lin, L., et al. (2017). Hydrogen sulfide reduces RAGE toxicity through inhibition of its dimer formation. Free Radic. Biol. Med. 104, 262-271. doi: 10.1016/j.freeradbiomed.2017. 01.026

Zhou, X., An, G., and Lu, X. (2015). Hydrogen sulfide attenuates the development of diabetic cardiomyopathy. Clin. Sci. 128, 325-335. doi: 10.1042/CS201 40460
Zou, F., Wang, L., Liu, H., Wang, W., Hu, L., Xiong, X., et al. (2019). Sophocarpine suppresses NF-кB-mediated inflammation both in vitro and in vivo and inhibits diabetic cardiomyopathy. Front. Pharmacol. 10:1219. doi: 10.3389/fphar.2019.01219

Conflict of Interest: The authors declare that the research was conducted in the absence of any commercial or financial relationships that could be construed as a potential conflict of interest.

Copyright (c) 2021 Zhao, Li, Li, Wei and Wang. This is an open-access article distributed under the terms of the Creative Commons Attribution License (CC BY). The use, distribution or reproduction in other forums is permitted, provided the original author(s) and the copyright owner(s) are credited and that the original publication in this journal is cited, in accordance with accepted academic practice. No use, distribution or reproduction is permitted which does not comply with these terms. 